\title{
Simultaneous human herpesvirus 6-associated encephalitis and Guillain-Barré syndrome in a patient after matched unrelated donor haematopoietic stem cell transplantation
}

Agnieszka Tomaszewska, Barbara Nasilowska-Adamska', Tomasz Dzieciatkowski², Bożena Marianska ${ }^{1}$

1Institute of Haematology and Transfusion Medicine, Warsaw, Poland

${ }^{2}$ Chair and Department of Medical Microbiology, Medical University of Warsaw, Warsaw, Poland

Submitted: 3 February 2009

Accepted: 29 March 2009

Arch Med Sci 2010; 6, 2: 288-290

DOI: 10.5114 /aoms.2010.13912

Copyright (c) 2010 Termedia \& Banach

\begin{abstract}
Viral infections are still a serious diagnostic and therapeutic problem in patients undergoing alternative donor transplants. $\beta$-Herpesviruses (especially human herpesvirus type 5, 6 and 7) are recognized pathogens in this group of patients and may cause central nervous system disease. Guillain-Barré syndrome (GBS) is a very rare complication among stem cell transplant recipients and usually has been attributed to infection. We report a case of resolving simultaneous GBS and HHV-6-associated encephalitis in a haematopoietic stem cell transplant recipient with preceding reactivation of cytomegalovirus (CMV) infection. According to our knowledge this well-documented case is probably the first report from Poland.
\end{abstract}

Key words: human herpesvirus-6, encephalitis, Guillain-Barré syndrome, haematopoietic stem cell transplant recipients.

\section{Introduction}

Human herpesvirus type 6 (HHV-6) belongs to the subfamily $\beta$-herpesviruses and is closely related to human herpesvirus type 7 ( $\mathrm{HHV}-7)$ and type 5 (HHV-5, formerly known as cytomegalovirus - CMV) [1]. All $\beta$-herpesviruses are emerging pathogens in haematopoietic stem cell transplant recipients (HSCT) and may cause central nervous system (CNS) disease $[1,2]$. Diagnosis and treatment of CMV infection is currently more advanced than for HHV-6 and HHV-7 [1, 3]. Guillain-Barré syndrome (GBS), an autoimmune acute peripheral neuropathy, is a very rare complication among stem cell transplant recipients and usually has been attributed to infection $[4,5]$. In this case report we present a patient with HHV-6 encephalitis complicated by GBS after matched unrelated donor stem cell transplantation with preceding reactivation of HHV-5 (CMV) infection. We also overview the treatment outcome in this patient. We received written informed consent from the patient for every invasive diagnostic procedure and for proposed therapy.

\section{Case report}

A 43-year-old man with a history of chronic myeloid leukaemia underwent HSCT from a matched unrelated female donor in October 2006

\section{Corresponding author:}

Agnieszka Tomaszewska Institute of Haematology and Transfusion Medicine Warsaw, Poland Phone: +48 223496360 Fax: +48 223496361 E-mail: agnieszka_tomaszewska@onet.eu 
after treosulfan-fludarabine-ATG conditioning. Serostatus for CMV was IgG positive in the recipient and IgG negative in his donor. On the $70^{\text {th }}$ posttransplantation day (PTD) the patient developed aGvHD (acute graft versus host disease) $11^{\circ}$ with skin involvement (generalised erythroderma) and was successfully treated with intravenous (IV) methylprednisolone [6]. On 8 March 2007 (the 149th PTD) he was admitted to our unit due to CMV infection reactivation (CMV DNA was $5523 \mathrm{geq} / \mathrm{ml}$ and $8041 \mathrm{geg} / \mathrm{ml}$ in 2 consecutive examinations). He started pre-emptive therapy with IV ganciclovir according to the EBMT (the European Group for Blood and Marrow Transplantation) standards [6, 7].

After 2 weeks of treatment he revealed high fever $\left(39.5^{\circ} \mathrm{C}\right)$, uroschesis (retention of urine), paraparesis, impaired consciousness and generalized epileptic seizure. Computed tomography (CT) of his brain was normal. A lumbar puncture revealed pleocytosis $(24 / \mu \mathrm{l})$ and elevated level of protein $(213.2 \mathrm{mg} / \mathrm{dl})$. Investigation of cerebrospinal fluid (CSF) by PCR (polymerase chain reaction) methods for infective causes of the patient's neurological decline including HSV t.1/2 (herpes simplex virus type 1/2), VZV (varicella-zoster virus), adenovirus, CMV and DNA Candida and Aspergillus and Mycobacterium tuberculosis were negative as well as CSF culture. Borreliosis and cryptococcosis were excluded too. But real-time PCR (quantitative commercial ALPCO MutaREAL HHV6 test) revealed in his CSF presence of HHV6 DNA (215 geq $/ \mathrm{ml})$. CMV DNA in blood samples was undetectable at this time. Detection of anti-HHV-6 specific antibodies in IgM and IgG classes was performed, using the commercial qualitative enzyme immunoassay (EIA) PanBio test. Our patient's HHV-6 serology was IgG positive in the blood and negative in the CSF (at the moment of diagnosis and after treatment) and IgM negative in every specimen. According to these findings and the neurological status of our patients we made a diagnosis of HHV6 encephalitis complicated by GBS. Therapy with foscarnet (all symptoms revealed during preemptive therapy with ganciclovir) and IVIG (intravenous immunoglobulins) was started. Due to GBS diagnosis we performed 5 procedures of plasmapheresis followed by physical rehabilitation. We observed gradual improvement in the neurological status of our patient, but on 18 April HHV-6 DNA was still detectable in control CSF examination (over $50 \mathrm{geq} / \mathrm{ml}$ ). Nuclear magnetic resonance (NMR) of our patient's brain was performed - without any abnormal findings. Negativisation of HHV-6 DNA in CSF was reached on 26 April - after 20 days of foscarnet therapy. After discharge the therapy was continued in the outpatient clinic with cidofovir given once a week for 4 weeks.
At present, 2 years after this episode, the patient remains in a good condition without CMV and HHV-6 reactivation, with only very slight neurological deficiency.

\section{Discussion}

Human herpesvirus type 6 is the aetiological agent of exanthema subitum, a febrile rash illness that can occur during early childhood [7]. After primary infection, HHV-6 remains latent except for periods of immunosuppression [7, 8]. Human herpesvirus type 6 reactivates in 33-48\% of patients who undergo HSCT and has been associated with CMV infection, pneumonitis, myelosuppression, encephalitis and hepatitis [5, 7, 8]. Human herpesvirus type 6 DNA is frequently detected in blood during the first months after transplantation in asymptomatic patients but not in CSF $[6,7]$. There are 2 variants of HHV- 6 A and B (HHV- 6 A/B), which differ from other human herpesviruses because of the unique ability of their genomes to integrate in a persistent latent state into chromosomes (it is HHV-6 chromosomal integration - HHV-6 Cl). Human herpesvirus type $6 \mathrm{Cl}$ does not represent active $\mathrm{HHV}-6$ infection and its prevalence in the population is described as $1-2 \%$. In our case we could not distinguish the HHV- 6 variant because the test used by us detects HHV-6 DNA without specification of type. Clinical course, negative results of HHV-6 DNA in blood and negativisation of HHV- 6 DNA in CSF of our patient allows us to exclude HHV-6 $\mathrm{Cl}$ and indicates HHV-6 B type as a cause of this case of encephalitis. It is known that HHV-7 is closely related to HHV-6 infection but its detection after HSCT is relatively infrequent [7]. In our case we performed the HHV-7 DNA detection test (major capsid protein [MCP] test) in the blood and CSF retrospectively (on frozen samples) results were negative at the time of diagnosis and after treatment. The only detected virus in the CSF of the presented patient was HHV-6.

Over the last 15 years several reports describing HHV-6-associated encephalitis in at least 48 HSCT recipients have been published $[2,7,9]$. But the association of HHV- 6 encephalitis and GBS in the HSCT setting has very limited observations [10]. Post-transplant GBS has usually been attributed to infection and very rarely to neurotoxicity of conditioning regimens $[4,10]$. Approximately $75 \%$ of patients show signs of infection shortly preceding diagnosis of GBS $[4,10]$. The detection of HHV-6 DNA in CSF is a strong indication that the virus is actively replicating within the CNS. An active HHV-6 infection in the CNS during the clinical course of GBS suggests that not only preceding but also concurrent infections might be associated with GBS [10].

According to our knowledge this welldocumented and successfully diagnosed and 
treated case of simultaneous GBS and HHV-6 encephalitis in a patient after HSCT is probably the first report from Poland. It is worth remembering that serology has no role in the diagnosis of HHV-6 infection in allo-SCT recipients; quantitative PCR for HHV-6 DNA is recommended for diagnosis of HHV-6 infection in peripheral blood or CSF $[7,11]$. Due to its extraordinary sensitivity and rapid performance, real-time PCR guarantees detection of virus reactivation even if there is a low number of virus copies present in the sample. Early detection of HHV-6 infection with molecular biology methods is favourable for the efficacy of antiviral chemotherapy [11]. Foscarnet or ganciclovir are recommended as first-line therapies for HHV-6 encephalitis after HSCT and cidofovir as a secondline treatment [7, 11]. In our case HHV-6 encephalitis occurred during pre-emptive therapy with ganciclovir due to CMV reactivation, which was a diagnostic challenge.

In conclusion, simultaneous HHV-6 encephalitis and GBS is a very uncommon complication after HSCT. Our well-documented and successfully treated case indicates that HSCT recipients with CNS signs and symptoms should have their CSF investigated for HHV-6; and other infectious agents should be excluded by applying prompt and appropriate diagnostic tools such as culture, microscopy and PCR, especially real-time PCR. Also, exclusion of the phenomenon of $\mathrm{HHV} 6 \mathrm{Cl}$ is required.

\section{References}

1. Ljungman P. Beta-Herpesvirus challenges in the transplant recipient. J Infect Dis 2002; 186 (Suppl 1): S99-109.

2. Zerr DM. Human herpesvirus 6 and central nervous system disease in hematopoietic cell transplantation. J Clin Virol 2006; 37 (Suppl 1): S52-6.

3. Ljungman P, Perez-Bercoff L, Jonsson J, et al. Risk factors for the development of cytomegalovirus disease afte allogeneic stem cell transplantation. Haematologica 2006; 91: 78-83.

4. Hughes RA, Cornblath DR. Guillain-Barré syndrome. Lancet 2005; 366: 1653-66.

5. Rodriguez V, Kuehnle I, Heslop HE, Khan S, Krance RA. Guillain-Barré syndrome after allogeneic hematopoietic stem cell transplantation. Bone Marrow Transpl 2002; 29: 515-7.

6. Apperley J, Carreras E, Gluckman E, Gratwohl A, Masszi T. Haematopoietic stem cell transplantation. The EBMT Handbook. The $5^{\text {th }}$ revised edition. Forum Service Editore, Genoa (Italy), 2008.

7. Ljungman $\mathrm{P}$, de la Camara R, Cordonnier $\mathrm{C}$, et al. Management of CMV, HHV-6, HHV-7 and Kaposi-sarcoma herpesvirus (HHV-8) infections in patients with hematological malignancies and after SCT. Bone Marrow Transpl 2008; 1-14.

8. Zerr DM, Corey L, Kim HW, Huang ML, Nguy L, Boeckh M. Clinical outcomes of human herpesvirus 6 reactivation after hematopoietic stem cell transplantation. Clin Infect Dis 2005; 40: 932-40.
9. Galvan M, Rotola A, Govoni V, Granieri E, Cassai E, Di Luca D. Simultaneous Guillain-Barré syndrome and active human herpesvirus 6 infection in the central nervous system. J Clin Virol 2007; 38: 271-2.

10. Holden SR, Vas AL. Severe encephalitis in a haematopoietic stem cell transplant recipient caused by reactivation of human herpesvirus 6 and 7. J Clin Virol 2007; 40: 245-7.

11. Pöhlmann C, Schetelig J, Reuner U, et al. Cidofovir and Foscarnet for treatment of human herpesvirus 6 encephalitis in a neutropenic stem cell transplant recipient. Clin Infect Dis 2007; 44: e118-20. 